\title{
Supraclinoid Internal Carotid Artery Fenestration Harboring an Unruptured Aneurysm and Another Remote Ruptured Aneurysm: Case Report and Review of the Literature
}

\author{
Seong-Ho Park, MD, Chang-Young Lee, MD \\ Department of Neurosurgery, Keimyung University School of Medicine, Daegu, Korea
}

\begin{abstract}
A fenestration of the supraclinoid internal carotid artery (ICA) is a rare congenital condition. We report an aneurysm arising from the proximal end of an ICA fenestration that was treated by endovascular coiling. Three-dimensional rotational angiography of preoperative cerebral angiography provided an understanding of the complex anatomy of the aneurysms associated with the fenestration and may facilitate the clinical decision regarding the treatment option. Endovascular coiling appears to be safe and effective for treating an aneurysm originating from a fenestration on the supraclinoid ICA, which is a difficult lesion to treat using a conventional surgical approach.
\end{abstract}

Keywords Internal carotid artery, Fenestration, Aneurysm, Coil embolization, Angiography

\author{
J Cerebrovasc Endovasc Neurosurg. \\ 2012 December; 14(4):295 299 \\ Received : 11 October 2012 \\ Revised : 12 November 2012 \\ Accepted : 4 December 2012 \\ Correspondence to Chang-Young Lee, MD \\ Department of Neurosurgery, Keimyung \\ University School of Medicine, 56 \\ Dalseong-ro, Jung-gu, Daegu, 700-712 \\ Republic of Korea \\ Tel : (001) 82-53-250-7732 \\ Fax : (001) 82-53-250-7356 \\ E-mail : nslcy@dsmc.or.kr
}

This is an Open Access article distributed under the terms of the Creative Commons Attribution NonCommercial License (http://creativecommons.org/licenses/by-nc/3.0) which permits unrestricted noncommercial use, distribution, and reproduction in any medium, provided the original work is properly cited.

\section{INTRODUCTION}

Intracranial arterial fenestration refers to segmental duplication of intracranial arteries, which is a rare congenital condition resulting from abnormal development of primitive embryologic vessels that occurs in $<0.7 \%$ of the population. ${ }^{16)}$ An arterial fenestration may harbor an aneurysm, presumably through the combination of hemodynamic stress and a medial defect. While almost all fenestrations are found in the vertebrobasilar system or anterior communicating region, the intracranial internal carotid artery (ICA) is an extremely rare site for fenestration. Moreover, a supraclinoid ICA fenestration harboring an aneurysm has been reported in relatively few cases. Treating an aneurysm associated with fenestration in this location is challenging for the neurosurgeon due to the anatomical complexity.

We describe an unruptured aneurysm associated with a fenestration of the supraclinoid ICA, which was successfully obliterated using endovascular coiling, and explain the pathogenesis and clinical features of supraclinoid ICA fenestrations and associated aneurysms in the cases reported in the literature.

\section{CASE REPORT}

A 44-year-old male presented with severe sudden-onset headache. Computed tomography (CT) scan showed dense blood clot in the left Sylvian fissure, suggesting ruptured middle cerebral artery (MCA) bifurcation aneurysm; CT angiography (CTA) revealed 


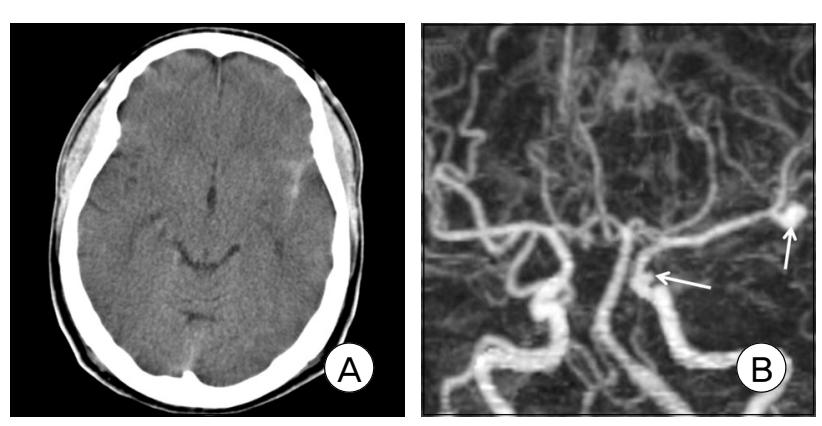

Fig. 1. (A) Noncontrast-enhanced computed tomography (CT) scan reveals a subarachnoid hemorrhage limited to the left Sylvian fissure. (B) CT angiography scan reveals aneurysms (arrows) at the left bifurcation of the middle cerebral artery (MCA) and left paraclinoid of the internal cerebral artery (ICA).

another supraclinoid ICA aneurysm as well as left MCA bifurcation aneurysm (Fig 1). For the treatment of ruptured MCA bifurcation aneurysm, considering the irregular shape, larger size, daughter sac and the location of the hematoma in preoperative CT scan, the patient underwent aneurysm clipping surgery via a left pterional craniotomy. A clip was successfully placed on the neck of the aneurysm, which was confirmed as ruptured in the surgical field. Although a paraclinoid aneurysm existed on the same side and could be clipped via the same approach, we planned endovascular treatment for the unruptured supraclinoid ICA aneurysm because of potential optic nerve injury attributable to anterior clinoidectomy or unroofing of the optic canal during clipping. The three-dimensional rotational angiography (3DRA) at 20 days after surgery demonstrated a fenestration of the left supraclinoid ICA with an aneurysm arising from its proximal end, which was not delineated clearly on 2D cerebral angiography and CTA, and the well-clipped state of the ruptured MCA bifurcation aneurysm. The fenestration of the ICA occurred from the origin of the ophthalmic artery (OphA) to the origin of the posterior communicating artery ( $\mathrm{PCoA})$. A saccular aneurysm was oriented postero-laterally, arising from the posterior aspect of the left supraclinoid ICA segment, which was proximal to the fenestration (Fig. 2). The aneurysm was 4.5, 3.4 and $3.5 \mathrm{~mm}$ in length, dome and neck diameter, respectively. From
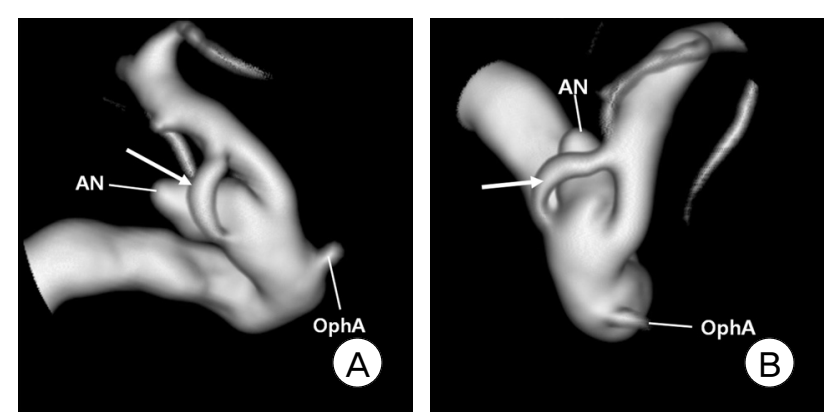

Fig. 2. (A and B) Three-dimensional rotational angiography (3DRA) shows any desired angle, and the complex vascular anatomy can be visualized. 3DRA reconstruction reveals the fenestration of the ICA with the associated aneurysm and the relationship between the cerebral arteries and the fenestration. AN, aneurysm; OphA, ophthalmic artery.

post-operative day 20, dual anti-platelet medication (aspirin $200 \mathrm{mg}$ and clopidogrel $75 \mathrm{mg}$ ) was administered for about seven days for the coil embolization of the supraclinoid ICA aneurysm. At 27 days after the surgical clipping, because of the wide-neck and vessel incorporation at the neck of the aneurysm, balloon-assisted endovascular coiling was performed to occlude the aneurysm and preserve both fenestration channels. Under local anesthesia, the $7 \mathrm{~F}$ femoral sheath was inserted through the right-sided femoral artery. Then the 7F guiding catheter (Vista Brite; Cordis Neurovascular, Miami, FL) was advanced to the distal cervical ICA segment. The Excelsior SL-10 microcatheter (Boston Scientific, Natick, MA) was placed into the neck of the aneurysm. Balloon-assisted coil embolization was done with Hyperglide
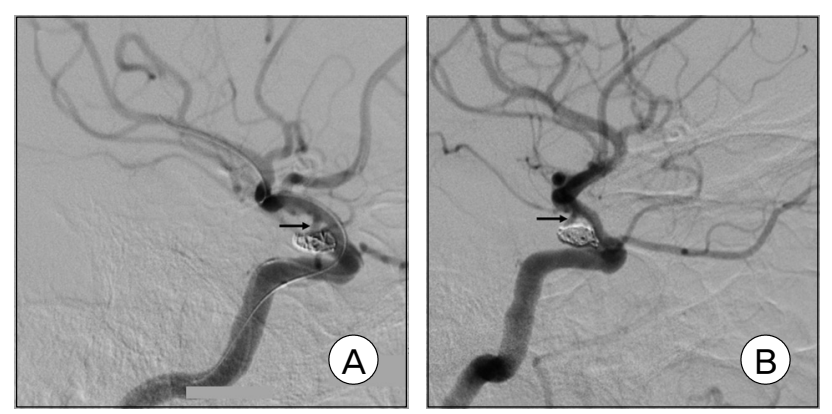

Fig. 3. (A) Working angle of digital subtraction angiography during coil embolization shows deploying coils under balloon assistance. (B) After coil embolization, complete occlusion was achieved with preservation of both fenestration channels. Arrows show the supraclinoid ICA fenestration. 
[Microtherapeutics (MTI), Irvine, CA]. We used five bare-platinum coils for the obliteration of the aneurysm. The aneurysm was obliterated successfully, and both limbs of the fenestration were preserved (Fig. 3). The patient had an uneventful postoperative course and was discharged without any neurological deficits.

\section{DISCUSSION}

The most common locations for intracranial artery fenestrations are the anterior communicating artery (AcoA), followed by the vertebrobasilar arteries, the anterior cerebral artery (ACA), and the MCA. ${ }^{6}$ Fenestrations of the intracranial ICA appear to be rare, with only 16 cases reported previously. 2)335-10)12-15)17720) A review of all literature on intracranial ICA fenestra- tion is summarized in Table 1. Fifteen of the 17 patients, including our case, had aneurysms at a fenestration or elsewhere. Twelve patients had aneurysms associated with a fenestration (proximal side only in nine, each trunk in one, large trunk in one, small trunk in one), and six aneurysms were apart from the fenestration in six patients (two AcoA, ICA terminal, contra-lateral PcoA, anterior choroidal artery [AchA], superior cerebellar artery, and MCA bifurcation). Only two of the 17 reported fenestration cases had no aneurysm anywhere within the intracranial arteries, ${ }^{3) 17}$ suggesting that an ICA fenestration is frequently associated with aneurysms at the fenestration or elsewhere. Fenestrations are widely believed to predispose patients to aneurysm formation. ${ }^{718)}$ However, if aneurysms could arise due to a combination of a defective arterial wall and altered hemodynamic

Table 1. Characteristics of supraclinoid ICA fenestration and associated aneurysms in the literature

\begin{tabular}{|c|c|c|c|c|c|c|}
\hline Author(year) & Age/Sex & Location & Aneurysm at fenestration & Remote Aneurysm & SAH & Treatment \\
\hline \multicolumn{7}{|c|}{ Fenestration without aneurysm } \\
\hline Takano et al. (1991) & $51 / \mathrm{F}$ & Right ICA & - & - & - & NA \\
\hline Bharatha et al. (2007) & $73 / \mathrm{M}$ & Right ICA & - & - & - & NA \\
\hline \multicolumn{7}{|c|}{ SAH from aneurysm associated with fenestration } \\
\hline Yock (1984) & $41 / \mathrm{F}$ & Right ICA & + (proximal) & - & + & Wrapping \\
\hline Chen et al. (2007) & $31 / \mathrm{M}$ & Bilateral ICA & + (right ICA large trunk) & - & + & Wrapping \\
\hline Case 2 of Nakiri et al. (2011) & $44 / M$ & Left ICA & $+; 2$ (same trunk) & - & + & Coil \\
\hline Case2 of Dey et al. (2011) & $32 / \mathrm{F}$ & Left ICA & + (proximal) & - & + & Clip \\
\hline \multicolumn{7}{|l|}{ SAH from remote aneurysm } \\
\hline Findlay et al. (1987) & $28 / \mathrm{F}$ & Right ICA & - & $+(A \operatorname{co} A)$ & + & NA \\
\hline Hattori et al. (1992) & $38 / F$ & Left ICA & - & + (left ICA terminal) & + & Clip \\
\hline Banach et al. (1993) & $37 / F$ & Left ICA & + (Proximal) & + (right PcoA) & + & Clip \\
\hline Katsuta et al. (1993) & $46 / F$ & $\begin{array}{l}\text { Right ICA/right } \\
\text { ACA Al }\end{array}$ & - & $+(\mathrm{SCA})$ & + & Wrapping \\
\hline Present Study & $44 / M$ & Left ICA & + (proximal) & + (left MCA) & + & Coil (ICA) /clip (MCA) \\
\hline \multicolumn{7}{|c|}{ Unruptured remote or fenestration associated aneurysm } \\
\hline $\mathrm{Ng}$ et al. (2006) & 34/F & Right ICA & $+; 2$ (each trunk) & - & - & Clip/coil \\
\hline Onoda et al. (2008) & $42 / \mathrm{F}$ & Left ICA & + (proximal) & - & - & Wrapping \\
\hline Plumb et al. (2010) & $48 / \mathrm{F}$ & Left ICA & + (proximal) & - & - & Clip \\
\hline Case 1 of Nakiri et al. (2011) & $47 / F$ & Right ICA & + (proximal) & - & - & Coil \\
\hline Case 1 of Dey et al. (2011) & 39/F & Right ICA & + (proximal) & - & - & Clip \\
\hline Ichikawa et al. (2011) & $47 / F$ & Left ICA & + (proximal) & + (left AchA) & - & Coil \\
\hline
\end{tabular}


stress, they would be expected to occur at the same rates in a fenestration and in branching points of the circle of Willis. ${ }^{116)}$ Although there is no clear correlation between fenestrations and aneurysms, we tentatively suggest that fenestrations at specific locations such as the supraclinoid ICA could be associated with aneurysms. Some authors ${ }^{1215) 19)}$ have also considered the possibility of an association between aneurysms and fenestrations in particular locations, such as the vertebral junction and supraclinoid ICA. The mechanism of the association between aneurysms and fenestrations at these specific locations is unknown, but we suggest that the incidence of development of an aneurysm may be increased in vertebral and supraclinoid ICA fenestrations due to relatively high hemodynamic stress because of their proximal location.

SAH occurred in nine of 15 patients with aneurysms. Some case reports suggested a relatively high incidence of SAH in patients who had an aneurysm with an ICA fenestration. . $^{8914}$ ) But because of small number of patients, this suggestion is somewhat controversial. Although fenestrations are associated with aneurysms, it is unproven that aneurysms associated with arterial fenestrations carry an increased risk for rupture compared with aneurysms arising from other sites, because more than half of the cases with a SAH involved a remote aneurysm.

The embryologic origin of this anomaly is not clearly understood due to its rarity. Normal development of the ICA arises from the third aortic arch at the 4to 5-mm embryonic stage when the primitive carotid artery divides to form the cranial and caudal divisions of the ICA. ${ }^{11)}$ The cranial division gives rise to the AchA, MCA, and ACA, whereas the caudal division terminates as the PcoA. Therefore, a supraclinoid ICA fenestration might be attributable to failure of the developing ICA to divide. Another possible etiology of an ICA fenestration is persistence of the small plexiform temporarily connected between two intracranial primitive carotid arteries at the 4- to 5-mm embryonic stage. ${ }^{2)}$ These theories would explain why all reported cases occurred just distal to the oph- thalmic artery, which corresponds to the division site of the primitive ICA. The fenestration in our patient also occurred from the origin of the OphA to the origin of the posterior PcoA. In a pathological study, Black et al. ${ }^{4)}$ identified a ventral defect in the muscularis layer of the vessel at each end of a fenestration and showed that an aneurysm arose at the proximal vessel wall defect. The combination of inherent structural wall weakness and local hemodynamic stress, particularly at a proximal site, may result in an aneurysm. ${ }^{2}$ In the previously reported cases and, in our patient, nine of the 14 aneurysms associated with a fenestration developed at the proximal end of the fenestration.

We performed CTA, magnetic resonance angiography (MRA), 2D angiography, and 3DRA as diagnostic tools for cerebral vascular lesions. Among them, 3DRA seems to be the best modality for evaluating a supraclinoid ICA fenestration with an associated aneurysm. The estimated angiographic prevalence of intracranial fenestrations in 2D angiography was $0.3-0.9 \% .{ }^{1618)}$ However, most fenestrations may be overlooked or be invisible because of over-lapping vessels on $2 \mathrm{D}$ angiography when compared with 3DRA, as demonstrated in a large retrospective study, which showed a prevalence of $28 \%$ fenestrations in 208 patients. Of 61 fenestrations detected on 3DRA, ten $(16 \%)$ were retrospectively visible on $2 \mathrm{D}$ angiographic images. ${ }^{19)}$ Chen et al. ${ }^{5)}$ reported the first conventional $2 \mathrm{D}$ angiography, showing the short segment of a fenestration of the bilateral supraclinoid ICA without associated aneurysms in the anterior circulation. But, repeated 3DRA showed a small aneurysm at the larger trunk of the right ICA fenestration.

Eight $(73 \%)$ of 11 patients with supraclinoid ICA aneurysm, in the previously reported papers were treated with open surgery. Among them, three were treated by wrapping and four by clipping. In one patient, who had two aneurysms, one aneurysm was treated by clipping and the other by endovascular coil embolization. Because of intracranial stenosis, blood 
flow of the fenestrated limb could not be maintained after direct clipping, so wrapping was done. ${ }^{14)}$ Direct surgical clipping, although successful, is extensively invasive with considerable potential risk related to aggressive anterior clinoidectomy or unroofing of the optic canal, whereas endovascular coiling avoids these destructive procedures and protects the patency of both fenestration channels using remodeling techniques under real-time visualization on angiographic monitoring during the entire procedure. When considering the difficulty of the surgical approach and potential surgical failure due to kinking of the parent artery, endovascular coiling seems appropriate to treat aneurysms associated with a supraclinoid ICA fenestration.

\section{CONCLUSION}

The 3DRA seemed to provide a clear delineation of the supraclinoid ICA fenestration compared to conventional imaging and accurately depicted the anatomy. Endovascular coiling appears to be a valid alternative to surgery for an aneurysm associated with a supraclinoid ICA fenestration to avoid surgical difficulties and failure.

\section{REFERENCES}

1. Ahn JH, Kim MS, Lee HK, Lee SJ, Park HI, Lee $\mathrm{CH}$. Fenestration and duplication of the vertebrobasilar artery detected by conventional angiography and magnetic resonance angiography. J Korean Neurosurg Soc. 2006 May; 39(5):355-9.

2. Banach MJ, Flamm ES. Supraclinoid internal carotid artery fenestration with an associated aneurysm. Case report. J Neurosurg. 1993 Sep;79(3):438-41.

3. Bharatha A, Fox AJ, Aviv RI, Symons SP. CT angiographic depiction of a supraclinoid ICA fenestration mimicking aneurysm, confirmed with catheter angiography. Surg Radiol Anat. 2007 Jun;29(4):317-21.

4. Black SP, Ansbacher LE. Saccular aneurysm associated with segmental duplication of the basilar artery. A morphological study. J Neurosurg. 1984 Dec;61(6):1005-8.

5. Chen YY, Chang FC, Hu HH, Chao AC. Fenestration of the supraclinoid internal carotid artery associated with aneurysm and ischemic stroke. Surg Neurol. 2007;68 Suppl 1(1):S60-3; discussion S63.
6. Dey M, Awad IA. Fenestration of supraclinoid internal carotid artery and associated aneurysm: embryogenesis, recognition, and management. World Neurosurg. 2011 Dec;76(6):592. e1-5.

7. Findlay JM, Chui M, Muller PJ. Fenestration of the supraclinoid internal carotid artery. Can J Neurol Sci. 1987 May;14(2):159-61.

8. Hattori T, Kobayashi H. Fenestration of the supraclinoid internal carotid artery associated with carotid bifurcation aneurysm. Surg Neurol. 1992 Apr;37(4):284-8.

9. Ichikawa $T$, Miyachi S, Izumi T, Matsubara N, Naito T, Haraguchi $\mathrm{K}$, et al. Fenestration of a supraclinoid internal carotid artery associated with dual aneurysms: case report. Neurosurgery. 2011 Oct;69(4):E1005-8; discussion E1009.

10. Katsuta T, Matsubara T, Fujii K. Fenestration of the supraclinoid internal carotid artery. Neuroradiology. 1993;35(6):461.

11. Lasjaunias P, Santoyo-Vazquez A. Segmental agenesis of the internal carotid artery: angiographic aspects with embryological discussion. Anat Clin. 1984;6(2):133-41.

12. Nakiri GS, Bravo E, Al-Khawaldeh M, Rivera R, Badilla L, Mounayer C. Endovascular treatment of aneurysm arising from fenestration of the supraclinoid internal carotid artery-two case reports. J Neuroradiol. 2012 Jul; 39(3):195-9.

13. Ng PP, Steinfort B, Stoodley MA. Internal carotid artery fenestration with dual aneurysms. Case illustration. J Neurosurg. 2006 Jun;104(6):979.

14. Onoda K, Ono S, Tokunaga K, Sugiu K, Date I. Fenestration of the supraclinoid internal carotid artery with associated aneurysm. Neurol Med Chir (Tokyo). 2008 Mar;48(3):118-20.

15. Plumb AA, Herwadkar A, Pickett G. Incidental finding of fenestration of the supraclinoid internal carotid artery with appearances on magnetic resonance angiography. Surg Radiol Anat. 2010 Feb;32(2):165-9.

16. Sanders WP, Sorek PA, Mehta BA. Fenestration of intracranial arteries with special attention to associated aneurysms and other anomalies. AJNR Am J Neuroradiol. 1993 May-Jun;14(3):675-80.

17. Takano S, Saitoh M, Miyasaka Y, Yada K, Takagi H. Fenestration of the intracranial internal carotid artery-case report. Neurol Med Chir (Tokyo). 1991 Nov; 31(11):740-2.

18. Teal JS, Rumbaugh CL, Bergeron RT, Segall HD. Angiographic demonstration of fenestrations of the intradural intracranial arteries. Radiology. 1973 Jan;106(1): 123-6.

19. van Rooij SB, van Rooij WJ, Sluzewski M, Sprengers ME. Fenestrations of intracranial arteries detected with 3D rotational angiography. AJNR Am J Neuroradiol. 2009 Aug;30(7):1347-50.

20. Yock DH Jr. Fenestration of the supraclinoid internal carotid artery with rupture of associated aneurysm. AJNR Am J Neuroradiol. 1984 Sep-Oct;5(5):634-6. 